ARTICLE

https://doi.org/10.1038/s41467-019-10263-4

\title{
Solar energy storage at an atomically defined organic-oxide hybrid interface
}

Christian Schuschke (D) 1, Chantal Hohner (1) 1, Martyn Jevric ${ }^{2}$, Anne Ugleholdt Petersen², Zhihang Wang ${ }^{2}$, Matthias Schwarz', Miroslav Kettner', Fabian Waidhas', Lukas Fromm³ ${ }^{3}$ Christopher J. Sumby ${ }^{4}$, Andreas Görling 3,5, Olaf Brummel ${ }^{1}$, Kasper Moth-Poulsen (1) ${ }^{2}$ \& Jörg Libuda (1) 1,5

Molecular photoswitches provide an extremely simple solution for solar energy conversion and storage. To convert stored energy to electricity, however, the photoswitch has to be coupled to a semiconducting electrode. In this work, we report on the assembly of an operational solar-energy-storing organic-oxide hybrid interface, which consists of a tailormade molecular photoswitch and an atomically-defined semiconducting oxide film. The synthesized norbornadiene derivative 2-cyano-3-(4-carboxyphenyl)norbornadiene (CNBD) was anchored to a well-ordered $\mathrm{CO}_{3} \mathrm{O}_{4}(111)$ surface by physical vapor deposition in ultrahigh vacuum. Using a photochemical infrared reflection absorption spectroscopy experiment, we demonstrate that the anchored CNBD monolayer remains operational, i.e., can be photoconverted to its energy-rich counterpart 2-cyano-3-(4-carboxyphenyl)quadricyclane (CQC). We show that the activation barrier for energy release remains unaffected by the anchoring reaction and the anchored photoswitch can be charged and discharged with high reversibility. Our atomically-defined solar-energy-storing model interface enables detailed studies of energy conversion processes at organic/oxide hybrid interfaces.

\footnotetext{
${ }^{1}$ Lehrstuhl für Physikalische Chemie II, Friedrich-Alexander-Universität Erlangen-Nürnberg, Egerlandstr. 3, 91058 Erlangen, Germany. ${ }^{2}$ Department of Chemistry and Chemical Engineering, Chalmers University of Technology, 41296 Gothenburg, Sweden. ${ }^{3}$ Lehrstuhl für Theoretische Chemie, FriedrichAlexander-Universität Erlangen-Nürnberg, Egerlandstr. 3, 91058 Erlangen, Germany. ${ }^{4}$ Department of Chemistry and the Centre for Advanced Nanomaterials, The University of Adelaide, Adelaide, South Australia 5005, Australia. ${ }^{5}$ Erlangen Catalysis Resource Center, Friedrich-Alexander-Universität ErlangenNürnberg, Egerlandstr. 3, 91058 Erlangen, Germany. Correspondence and requests for materials should be addressed to J.L. (email: joerg.libuda@fau.de)
} 
T he development of new technologies for solar-energy conversion and storage is among the grand challenges in our transition to a renewable energy system ${ }^{1,2}$. Besides the conventional technologies, alternative chemical methods can provide particularly simple solutions for long-term solar-energy storage. Among these methods is energy storage in molecular photoswitches ${ }^{3-5}$, such as the valence couple norbornadiene (NBD)/quadricyclane $(\mathrm{QC})^{6-15}$. In this storage couple, the energy-lean NBD photoisomerizes via an intermolecular cycloaddition to yield the energy-rich QC. This extremely simple one-photon-one-molecule reaction allows storing up to $89 \mathrm{~kJ} / \mathrm{mol}$ $(0.97 \mathrm{MJ} / \mathrm{kg})$ of chemical energy, an energy density that is comparable with state-of-the-art batteries.

The NBD-QC system has been proposed to hold great potential for solar-energy harvesting and storage; however, it also comes with a number of challenges. Recently, several of these challenges, which were encountered in earlier research, could be successfully addressed and the system attracted renewed attention $^{12,13}$.

One issue is related to the fact that pristine NBD absorbs light at wavelengths below $300 \mathrm{~nm}$ only. Therefore, photosensitizers are required to red-shift the absorption ${ }^{8,16}$. These photosensitizers, however, cause stability issues, not only during photoconversion but also during the catalytically triggered energy release. Typically, this problem is tackled by using substituted NBDs that absorb light at much larger wavelength ${ }^{17,18}$. Such modifications, however, come at the price of increasing the molecular weight and, thereby, decreasing the energy density. Some authors of this work recently suggested a variety of compounds that combine beneficial absorption properties and low molecular weight ${ }^{18}$. Alternatively, the energy density can be increased by attaching more than one NBD unit to a single chromophore ${ }^{19}$. A second issue is related to the fact that redshifting the absorption maximum of NBD often destabilizes the corresponding QC isomer. However, this challenge could also be addressed by molecular design ${ }^{20,21}$. Recently, macroscopic heat release from a molecular solar thermal (MOST) storage device was demonstrated with a reversibility of more than $99.8 \%$ per storage cycle ${ }^{13}$. The energy release was triggered catalytically using a carbon-supported cobalt phthalocyanine catalyst.

A particularly fascinating idea is to control the NBD/QC storage system electrochemically ${ }^{22}$. Recently, we have shown that the back conversion can indeed be triggered electrochemically with a reversibility of $95 \%$, even in the presence of an external photosensitizer $^{23}$. The electrochemical approach does not only provide additional control but also holds the potential of converting part of the stored energy directly to electricity ${ }^{22}$. In principle, this may enable the construction of an energy-storing solar cell; however, the NBD unit would have to be coupled to a semiconducting electrode, for instance by attaching it via suitable anchor groups (similar as in a dye-sensitized solar cell).

While anchored NBD films could be prepared recently ${ }^{24}$, a functioning energy-storing hybrid interface, consisting of a photoswitchable NBD monolayer bound to an oxide surface, has not been reported to date. In this work, we demonstrate photochemical switching in an anchored NBD monolayer bound to an atomically defined oxide surface.

We used a synthesized NBD derivative that features four functionalities (see Fig. 1): (i) an NBD energy-storage unit, (ii) push-pull ligands that red-shift the absorption into the nearvisible region, (iii) a spectroscopic marker that allows monitoring the conversion by in-situ IR spectroscopy, and (iv) a carboxylic acid linker group for attachment to the oxide. The NBD derivative is anchored to an atomically defined $\mathrm{Co}_{3} \mathrm{O}_{4}(111)$ film with a known surface structure ${ }^{25,26}$. Both the assembly of the hybrid interface and the photochemical energy storage experiments were performed under ultraclean conditions in ultrahigh vacuum (UHV).

Our work demonstrates that it is possible to build an operational solar-energy-storing hybrid interface that consists of a single-anchored NBD monolayer on a well-defined semiconducting oxide surface. The interface is stable and photoswitchable with high reversibility. Our hybrid interface represents a type of model system, in which both the anchored film and the oxide surface are well defined at the atomic level. We believe that the type of model system described herein will enable studies of energy storage and release processes at such interfaces at a high level of detail.

\section{Results}

Preparation and properties of the model interface. The photoswitchable NBD monolayer was prepared from the NBD derivative 2-cyano-3-(4-carboxyphenyl)norbornadiene (CNBD) shown in Fig. 1a (see the Methods section for synthesis and Supplementary Methods for properties). The molecule comprises four essential functionalities: (i) the NBD storage unit, (ii) the donor-acceptor substituent pair which red-shifts the light absorption region, (iii) the CN group which is used as a marker for IR spectroscopy (see below), and (iv) the carboxylate group which acts as an anchor for oxide surfaces. Upon irradiation, CNBD converts to its energy-rich counterpart 2-cyano-3-(4-carboxyphenyl)quadricyclane (CQC) storing $0.363 \mathrm{MJ} / \mathrm{kg}$ in form of chemical energy (see Supplementary Methods). CNBD shows an absorption onset at $378 \mathrm{~nm}$ and absorption maximum at $319 \mathrm{~nm}$, while CQC absorbs at much shorter wavelengths (see Supplementary Methods). Therefore, irradiation with ultraviolet (UV) light leads to nearly quantitative conversion of CNBD to CQC.

CNBD films were deposited by physical vapor deposition (PVD) in UHV onto an ordered $\mathrm{Co}_{3} \mathrm{O}_{4}(111)$ film (see Fig. 1b). The $\mathrm{Co}_{3} \mathrm{O}_{4}(111)$ films (thickness $8 \mathrm{~nm}$ ) were grown on an $\operatorname{Ir}(100)$ single crystal using the procedure introduced by Heinz, Hammer et al. ${ }^{27}$. The surface of the $\mathrm{Co}_{3} \mathrm{O}_{4}(111)$ film has been characterized in detail by scanning tunneling microscopy (STM) and lowenergy electron diffraction I-V analysis (LEED-IV). It is terminated by a layer of $\mathrm{Co}^{2+}$ ions in the tetrahedral positions of the $\mathrm{Co}_{3} \mathrm{O}_{4}$ spinel structure (see Fig. 1a). Note that bulk $\mathrm{Co}_{3} \mathrm{O}_{4}$ is a semiconducting oxide with a bandgap of $1.6 \mathrm{eV}^{28}$, while STM studies suggest that the bandgap is larger for the thin films used in this work ${ }^{26}$. In order to verify that CNBD can be evaporated without decomposition, we deposited a multilayer film onto $\mathrm{Co}_{3} \mathrm{O}_{4}(111)$ and compared the infrared reflection absorption spectrum to the spectrum recorded in transmission (see Fig. 1c). Identical bands are found in both spectra, indicating that the deposition of a pure CNBD layer is possible without any decomposition products detected (note that the splitting of the carboxyl stretching band at $1700 \mathrm{~cm}^{-1}$ in IRAS indicates dimer formation on the frozen multilayer).

In order to identify the vibrational bands, we performed density functional theory (DFT) calculations of the CNBD dimer and analyzed the corresponding modes (see Supplementary Methods). The experimental and theoretically calculated band positions and their assignments are given in Supplementary Table 1. For this work, the most important feature is the $\mathrm{CN}$ stretching band $v_{\mathrm{CNBD}}(\mathrm{CN})$ at $2204 \mathrm{~cm}^{-1}$ (DFT $\left.2209 \mathrm{~cm}^{-1}\right)$. After photoconversion to CQC, the band blue-shifts to $2225 \mathrm{~cm}^{-1}\left(v_{\mathrm{CQC}}(\mathrm{CN})\right.$, DFT $2239 \mathrm{~cm}^{-1}$ ). This shift can be clearly resolved by IR spectroscopy, and will be used in this work to follow the interconversion between the two isomers. The complete IR spectra of CQC recorded in reflection and transmission modes and the spectrum calculated by DFT are shown in Fig. 1d. The band positions and assignments are also given in Supplementary Table 1. 
a
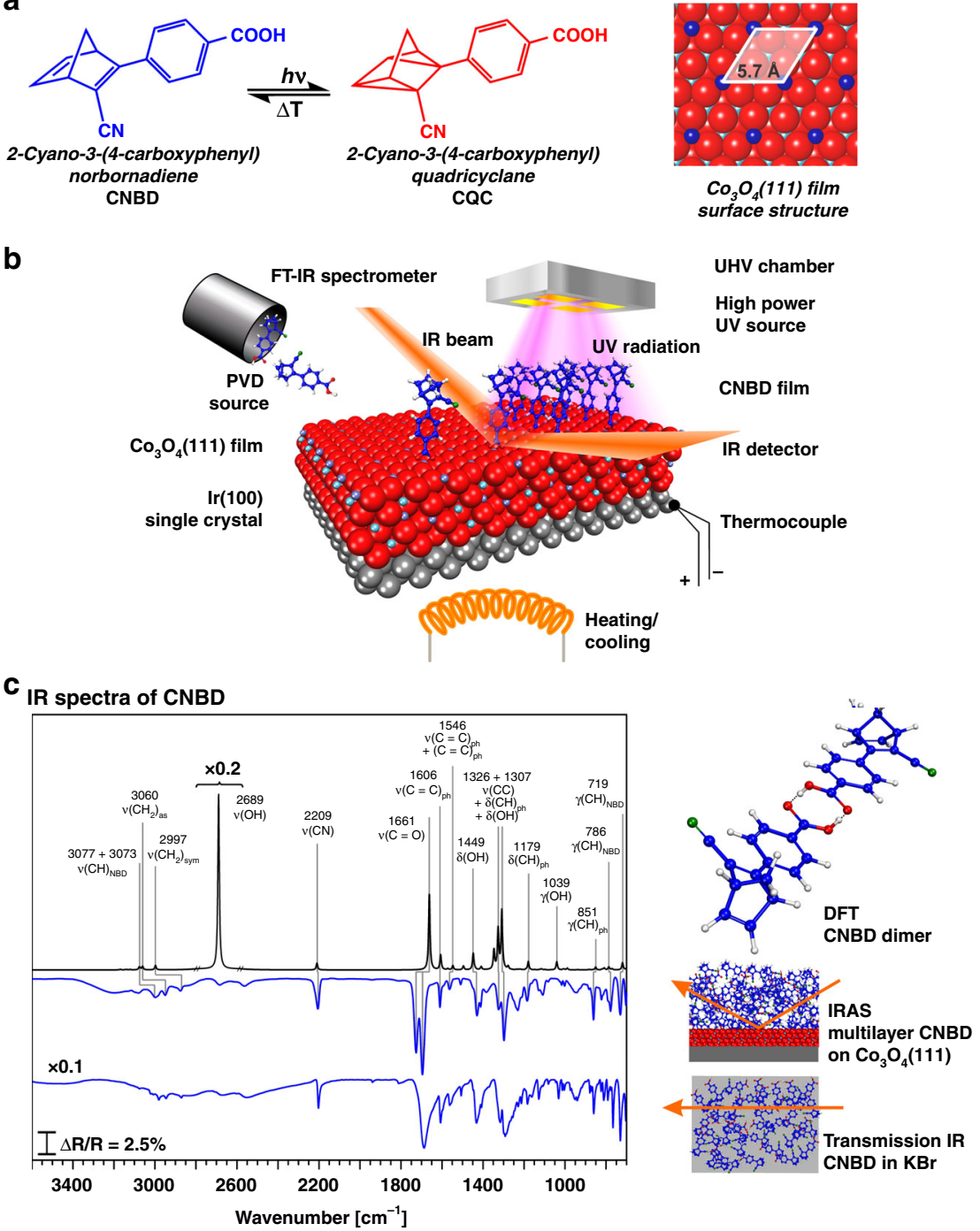

\section{$\mathbf{d}_{\text {IR spectra of CQC }}$}
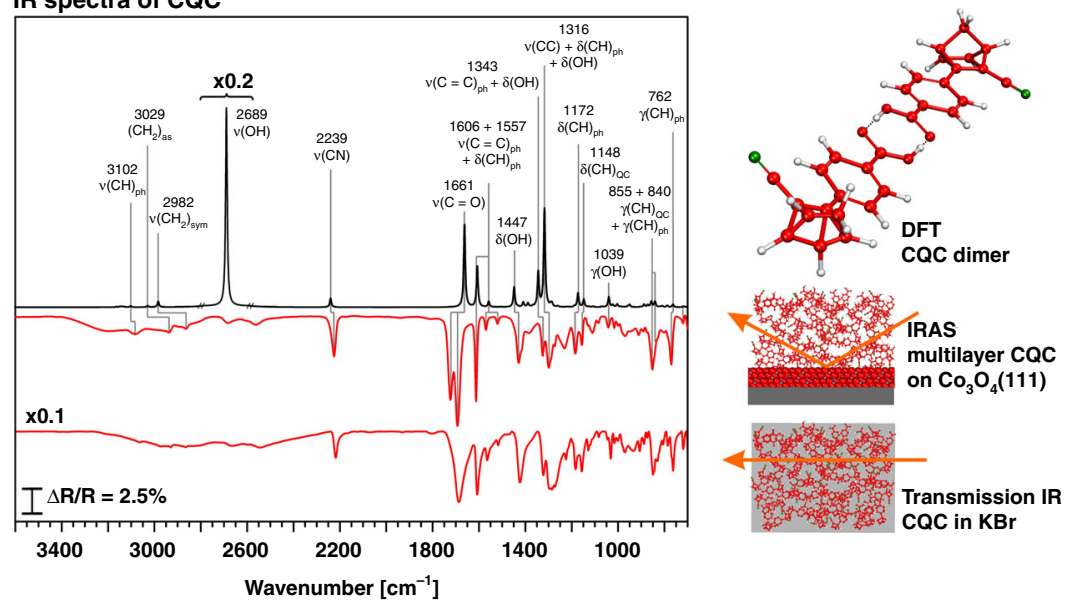

Fig. 1 The molecular photoswitch used in this study: a molecular structures of the energy-lean isomer CNBD and the energy-rich isomer CQC; b schematic representation of the in-situ photochemical IRAS setup in UHV used in this work; c IR spectra of CNBD (from top to bottom): IR spectrum calculated by DFT, IR reflection absorption spectrum of CNBD multilayer deposited by PVD referenced to a background of a clean sample, IR transmission spectrum of CNBD in $\mathrm{KBr}$; $\mathbf{d}$ IR spectra of CQC (from top to bottom): IR spectrum calculated by DFT, IR reflection absorption spectrum of CQC multilayer deposited by PVD referenced to a background of a clean sample, IR transmission spectrum of CQC in $\mathrm{KBr}$ 
Growth of CNBD films on $\mathrm{Co}_{3} \mathrm{O}_{4}(\mathbf{1 1 1})$. In the next step, we investigated the growth of CNBD films on $\mathrm{Co}_{3} \mathrm{O}_{4}(111)$. In Fig. 2a, infrared reflection absorption spectra are displayed, which were recorded during the deposition of a multilayer film at $110 \mathrm{~K}$. At the initial stage of deposition, the IR spectra are dominated by a broad band at $1400 \mathrm{~cm}^{-1}$, while the $v(\mathrm{C}=\mathrm{O})$ band of the carboxylic acid at $1700 \mathrm{~cm}^{-1}$ is the most intense feature at larger exposure. The band at $1400 \mathrm{~cm}^{-1}$ is attributed to the symmetric

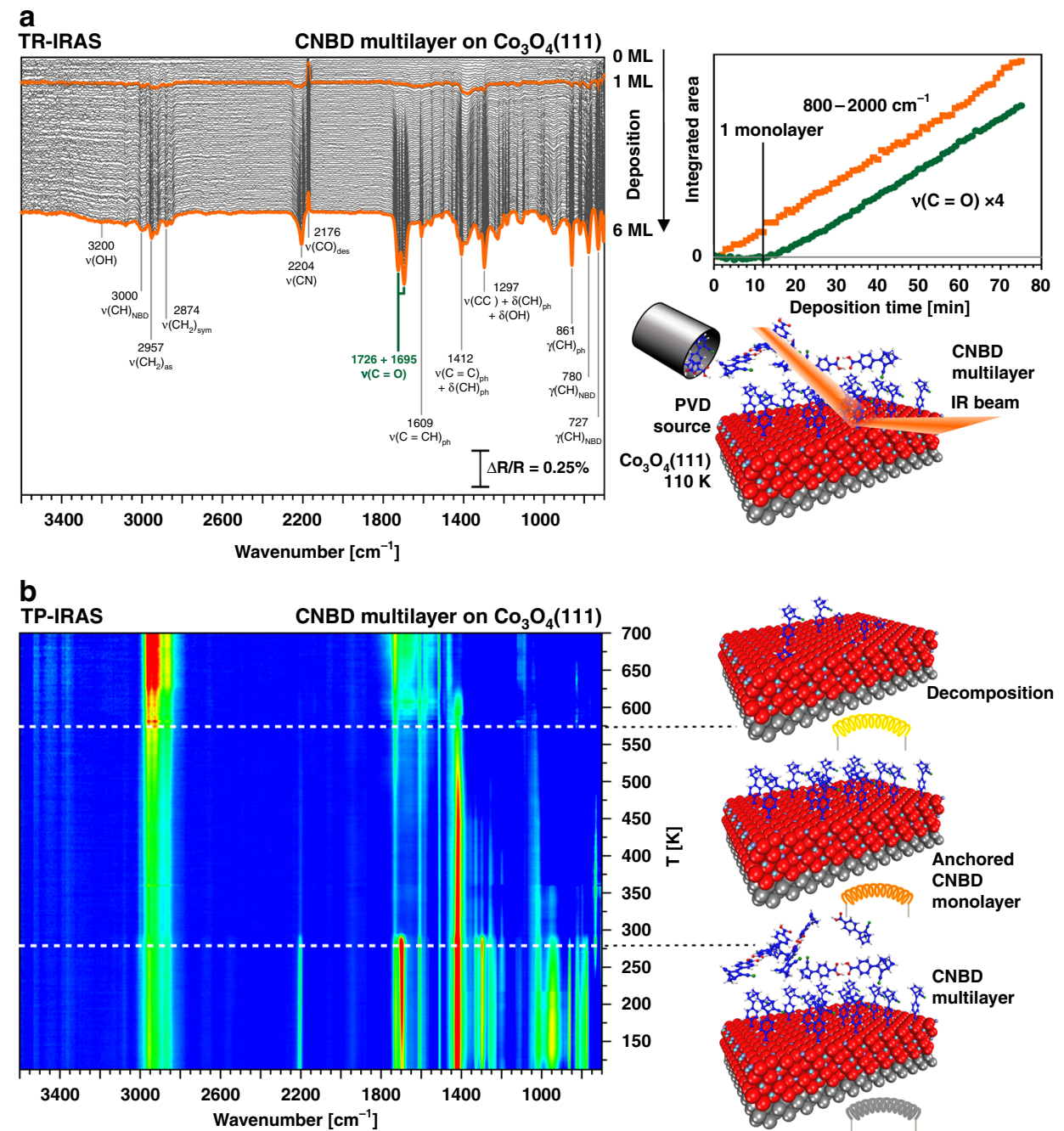

C

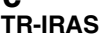

CNBD monolayer on $\mathrm{Co}_{3} \mathrm{O}_{4}(111)$
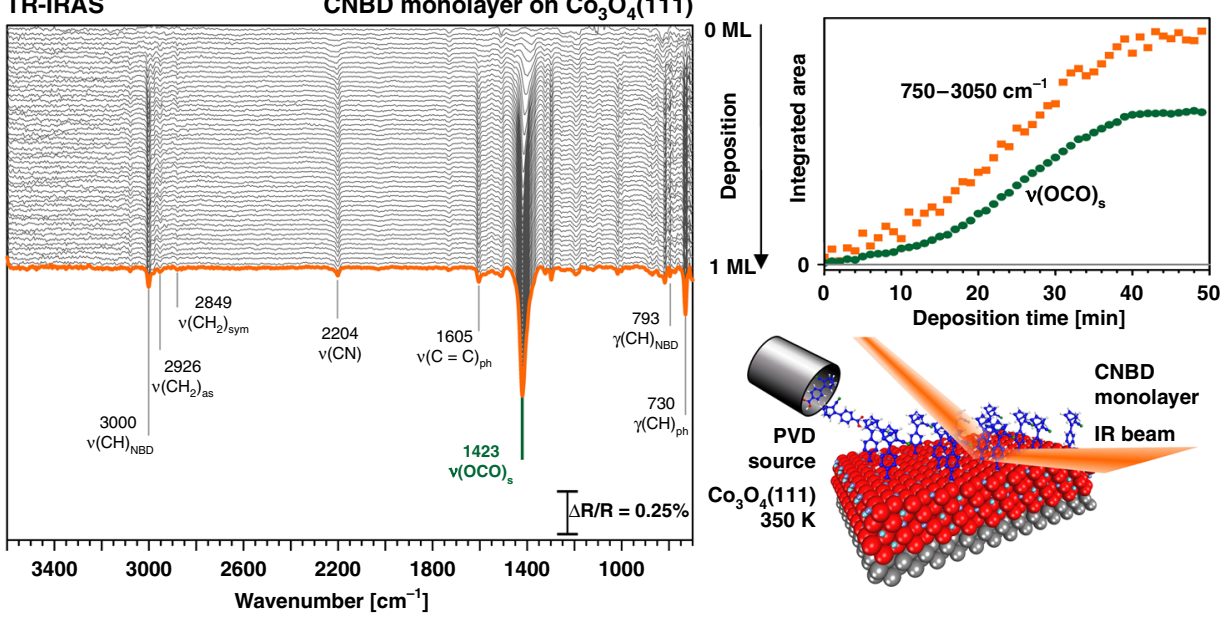

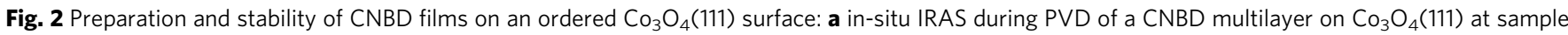
temperature $110 \mathrm{~K}$ (orange squares: total intensity, green circles: intensity of $\nu(C=O)$ band of $C N B D)$; $\mathbf{b}$ temperature-programmed IRAS after deposition of a CNBD multilayer showing the stability regions of the multilayer and monolayer; c preparation of an anchored monolayer by PVD of CNBD onto $\mathrm{CO}_{3} \mathrm{O}_{4}(111)$ at a sample temperature of $350 \mathrm{~K}$, i.e. above the multilayer desorption temperature (orange squares: the total intensity, green circles: intensity of $\nu(O C O)_{s}$ band of anchored $\left.C N B D\right)$. All spectra were referenced to the clean sample 
stretching mode $v_{s}(\mathrm{OCO})$ of a surface-bound carboxylate $\mathrm{e}^{24,29,30}$. It indicates that $\mathrm{CNBD}$ anchors to the surface by deprotonation and formation of a chelating surface carboxylate that is attached to the surface $\mathrm{Co}^{2+}$ ions. After completion of the anchored monolayer, a multilayer of non-anchored CNBD starts to grow, as indicated by the appearance of the $v(\mathrm{C}=\mathrm{O})$ band of the CNBD (see inset in Fig. 2a).

The thermal behavior of the CNBD multilayer film was probed by temperature-programmed (TP) IRAS, as shown in Fig. $2 \mathrm{~b}$. Here, IR spectra were recorded continuously, while the film was heated at a constant rate of $2 \mathrm{~K} \cdot \mathrm{min}^{-1}$. We observe a sudden decrease of all bands of the free CNBD at $280 \mathrm{~K}$, which we attribute to desorption of the multilayer. The characteristic $v_{s}(\mathrm{OCO})$ band of the surface-bound carboxylate is visible up to $570 \mathrm{~K}$, indicating that the anchored CNBD monolayer resides on the surface up to this temperature. At higher temperature, several weaker bands are observed, which we attribute to decomposition products of CNBD formed at higher temperature. The behavior is consistent with other carboxylate films, studied previously on the same surface 24,31 .

As the anchored CNBD monolayer is stable between $280 \mathrm{~K}$ and $570 \mathrm{~K}$, it should be possible to prepare a pure anchored monolayer film by PVD at surface temperatures in this range. The corresponding experiment is shown in Fig. $2 c$, where CNBD was deposited at a sample temperature of $350 \mathrm{~K}$. All bands show a saturation behavior, with the $v_{\mathrm{s}}(\mathrm{OCO})$ band of the surface carboxylate being the dominating feature. We conclude that an anchored monolayer is formed. No indication is observed for the adsorption of CNBD molecules that are not anchored to the surface.

Photochemical conversion of CNBD. In the next step, we investigated the photoconversion of CNBD films using a photochemical UHV IRAS setup that was recently developed by some of the authors (see Fig. 1b). The experimental procedure is illustrated in Fig. 3a. The film was exposed to exponentially increasing doses of UV light (irradiation times: $0.01 \mathrm{~s}, 0.04 \mathrm{~s}$, $0.16 \mathrm{~s}, \ldots, 655.36 \mathrm{~s}$ ), with each step followed by the acquisition of an IR spectrum. We used a UV source with a wavelength of $365 \mathrm{~nm}$ with an estimated power density of $920 \mathrm{~mW} \cdot \mathrm{cm}^{-2}$ at the sample surface ${ }^{32}$. The development in the spectral region of the $v$ $(\mathrm{CN})$ band is shown in Fig. 3b for a thick multilayer film (140 monolayer equivalents, ML), a thin multilayer film (6 ML), and a single-anchored monolayer $(1 \mathrm{ML})$. In all cases, we observe the disappearance of the $v_{\mathrm{CNBD}}(\mathrm{CN})$ band at $2204 \mathrm{~cm}^{-1}$ and the appearance of the $v_{\mathrm{CQC}}(\mathrm{CN})$ band at $2225 \mathrm{~cm}^{-1}$ upon irradiation, clearly showing that conversion of CNBD to CQC is possible, both in the multilayer and in the monolayer regimes.

Next, the photochemical conversion was analyzed quantitatively. We show three quantities that were calculated from the above spectra: the fraction of CNBD remaining as a function of irradiation time (Fig. 3c), the external quantum yield, i.e., the number of converted CNBD molecules per incident photon (Fig. 3d), and the photoconversion probability per molecule (Fig. 3e; See Supplementary Discussion for details).

The data show that the photoconversion reaction in the CNBD films strongly depends on two experimental parameters, the film thickness and the fraction of converted CNBD. The photoconversion is the fastest for thick CNBD films and for low conversion levels.

In order to unravel the origin of these dependencies, we consider the conversion in the thick multilayer in more detail. The reaction probability per molecule (see Fig. 3e) decreases rapidly with increasing conversion. Close to full conversion, it finally becomes very low and the uncertainty becomes large because of the low concentration of residual CNBD. One effect that contributes to this decrease is the different orientation of the CNBD molecules with respect to the electric field of the incident UV light. A quantitative analysis of this orientation effect (in which we assume random orientation in the multilayer) shows, however, that this effect only leads to a coverage dependence, which is much weaker than one observed experimentally (see Supplementary Discussion for details). Therefore, we suggest that the coverage dependence is mainly caused by support effects, i.e., by the underlying $\mathrm{Co}_{3} \mathrm{O}_{4}(111)$ substrate. Support effects can reduce the conversion probability, for example by quenching of excited CNBD through energy transport to the support or by generation of hot electrons and electron-hole pairs, which trigger the backconversion of CQC to CNBD. Previously, we have observed a similar substrate effect in condensed films of non-functionalized $\mathrm{NBD}^{32}$.

Interestingly, the initial photoconversion probability also decreases with decreasing film thickness (see Fig. 3e). Experimentally, we observe a decrease by a factor of $40( \pm 50 \%)$ between the $140 \mathrm{ML}$ and the $1 \mathrm{ML}$ films. This effect may be caused by the preferential molecular orientation in the monolayer, intermolecular interactions which modify the absorption spectrum, and screening of the electric field of the incident UV light at the metallic Ir substrate. A quantitative analysis of this electric-field effect shows that variations of the conversion rate by up to a factor of 30 are possible throughout a sufficiently thick film (see Supplementary Discussion for details). Therefore, it is likely that the electric-field effect largely contributes to the variation of the initial photoconversion probability, along with the support effects mentioned above. The importance of the latter is illustrated by the strong decrease of the photoconversion probability for the monolayer film, with increasing conversion (see Fig. 3e). Here, the conversion probability decreases by 2 orders of magnitude between $0 \%$ and $60 \%$ conversion. As molecular orientation effects typically play a lesser role in the anchored monolayer (where the molecules commonly adopt a similar orientation, in contrast to the randomly oriented multilayer), we assume that the decrease in the transition probability is mainly caused by support effects (i.e., by quenching or backconversion to CNBD).

Thermally activated backconversion. In the next step, we investigated the thermally activated backconversion of the anchored CQC monolayer. The experimental procedure is illustrated in Fig. 4a, b. After preparation of the CNBD monolayer, the film was exposed to a UV pulse (60 s), after which the thermally activated backconversion was recorded by time-resolved IRAS.

As the monolayer IR bands are extremely weak $(\Delta R / R \sim 0.01 \%)$, the photoconversion-decay cycles were repeated for $15 \mathrm{~h}$ and the corresponding IR data were accumulated. Such isothermal photoconversion-decay measurements were performed at temperatures between 350 and $370 \mathrm{~K}$.

The experimental data are shown in Fig. 4c (right panel) in form of IR difference spectra (see the Methods section for details). The decrease of the negative $v_{\mathrm{CQC}}(\mathrm{CN})$ band at $2225 \mathrm{~cm}^{-1}$ and the increase of the positive $v_{\mathrm{CNBD}}(\mathrm{CN})$ band at $2204 \mathrm{~cm}^{-1}$ as a function of time clearly proves that anchored CQC is thermally back-converted to CNBD. With increasing sample temperature, we observe that the rate of backconversion becomes faster (Fig. 4c, right panel). The data allow us to derive the activation energy for thermally activated backconversion in the anchored CQC monolayer (see Fig. 4c), yielding a value of $103 \pm 10 \mathrm{~kJ} \cdot \mathrm{mol}^{-1}$. In order to investigate whether the anchoring reaction has an effect on the backconversion, we also measured the kinetics of the 
a

Photochemical conversion - in-situ IRAS experiment

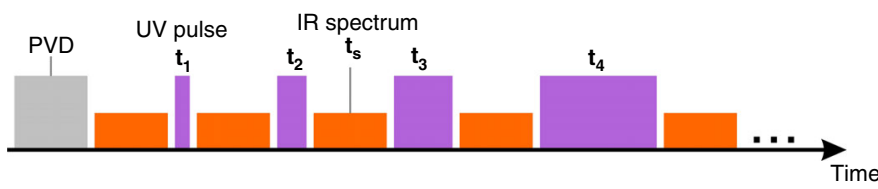

b

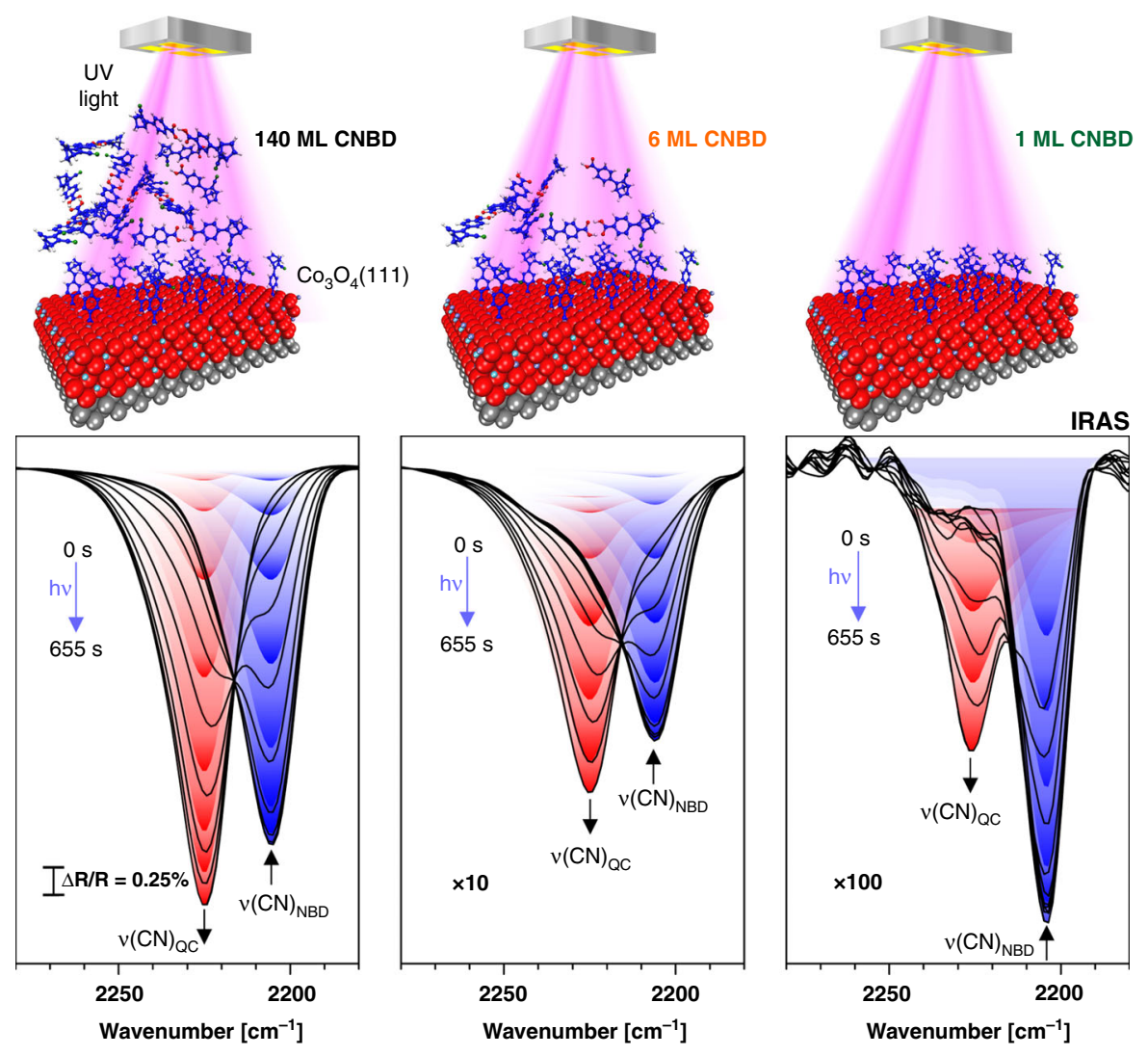

C

d
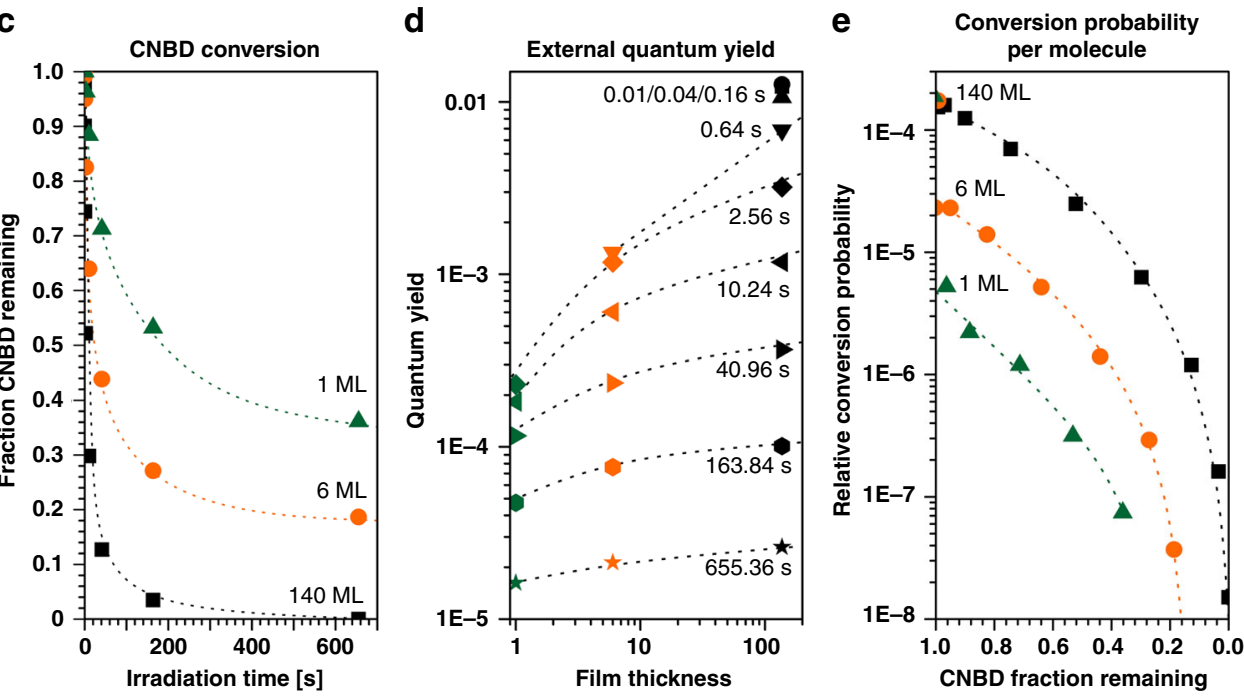

Fig. 3 Photochemical conversion of $\mathrm{CNBD}$ films on an ordered $\mathrm{Co}_{3} \mathrm{O}_{4}(111)$ surface: a the experimental procedure used in the photochemical IRAS experiment; $\mathbf{b}$ spectral region of the CN stretching bands of CNBD and CQC during UV irradiation for a thick multilayer film (140 ML), a thin multilayer film $(6 \mathrm{ML})$, and an anchored monolayer film (1 ML). All spectra were referenced to the clean sample; $\mathbf{c}$ the fraction of residual CNBD as a function of irradiation time (green triangles: $1 \mathrm{ML}$, orange circles: $6 \mathrm{ML}$, black squares: $140 \mathrm{ML}$ ); d external quantum efficiency as a function of film thickness and irradiation time (circles: $0.01 \mathrm{~s}$, squares: $0.04 \mathrm{~s}$, triangles (top): $0.16 \mathrm{~s}$, triangles (bottom): $0.64 \mathrm{~s}$, diamonds: $2.56 \mathrm{~s}$, triangles (left): $10.24 \mathrm{~s}$, triangles (right): $40.96 \mathrm{~s}$, hexagons: $163.84 \mathrm{~s}$, stars: $655.36 \mathrm{~s}$ ); e photoconversion probability per molecule as a function of conversion and film thickness (green triangles: $1 \mathrm{ML}$, orange circles: $6 \mathrm{ML}$, black squares: $140 \mathrm{ML} \mathrm{s}$ ) 
a Thermally activated backconversion - in-situ IRAS experiment $m$ cycles (total time $t_{3}$ )

b
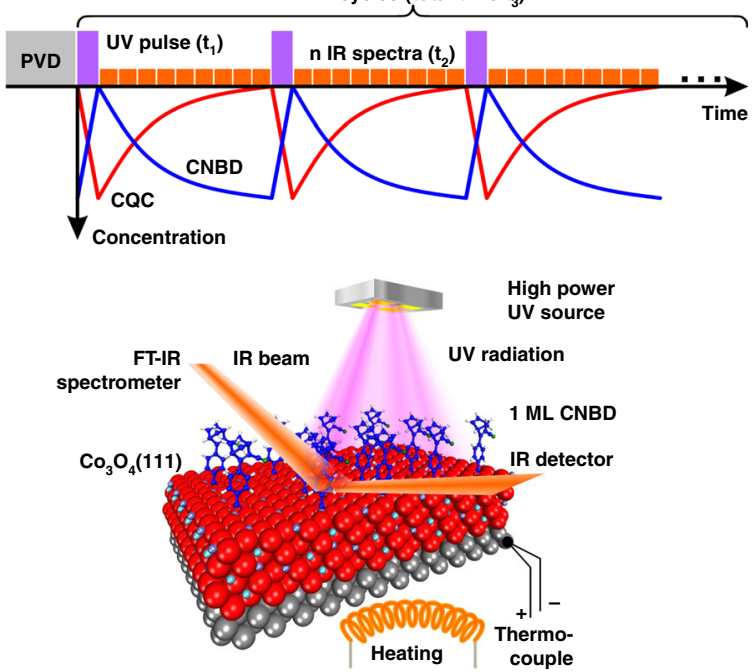

C

TR-IRAS

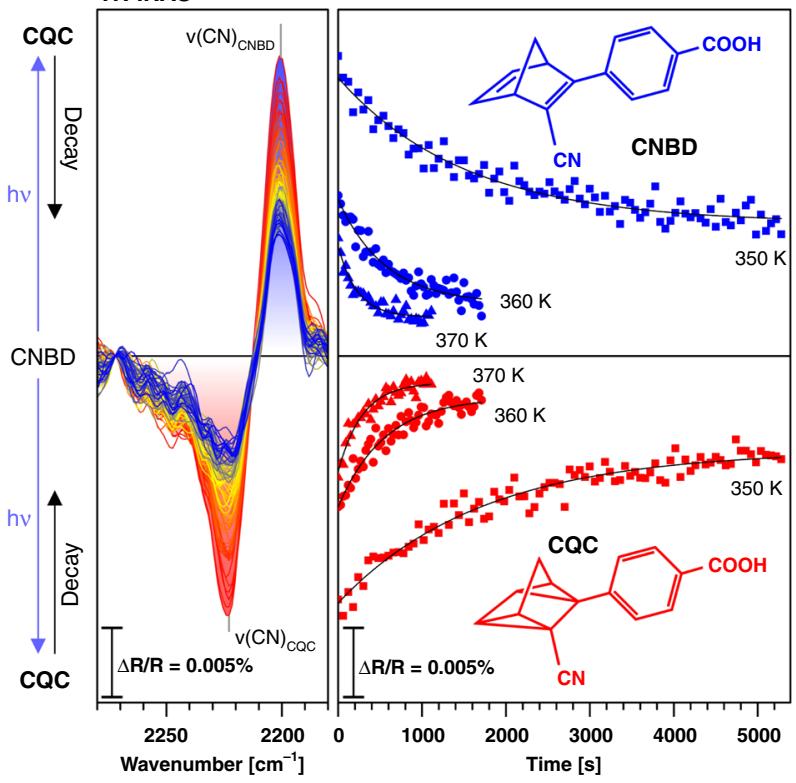

d

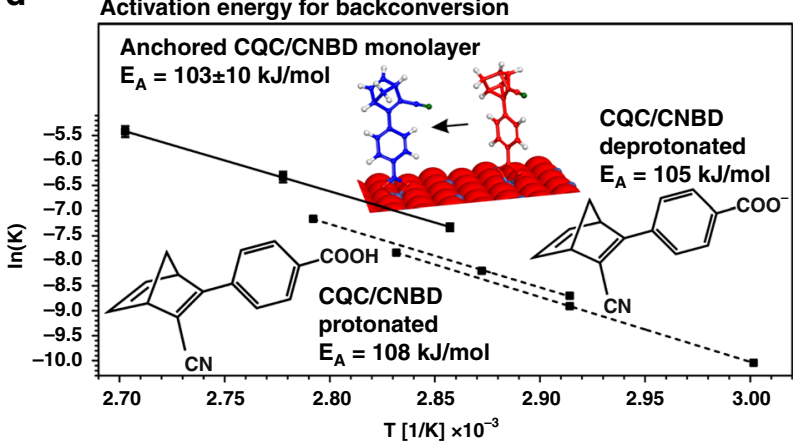

Fig. 4 Thermally activated backconversion of a CNBD monolayer on $\mathrm{Co}_{3} \mathrm{O}_{4}(111)$ : $\mathbf{a}$ the experimental procedure and (b) experimental setup used in the in-situ IRAS experiment; c spectra and intensities of the $\mathrm{CN}$ stretching bands of CNBD and CQC during photochemical conversion and thermally activated backconversion, referenced to the anchored CNBD monolayer (squares: $350 \mathrm{~K}$, circles: $360 \mathrm{~K}$, triangles: $370 \mathrm{~K}$ ); d Arrhenius plot comparing the kinetic parameters for backconversion of the anchored CNBD monolayer and free CNBD in solution (protonated and deprotonated form) (anchored: connected by straight line, in solution: connected by dashed line) a

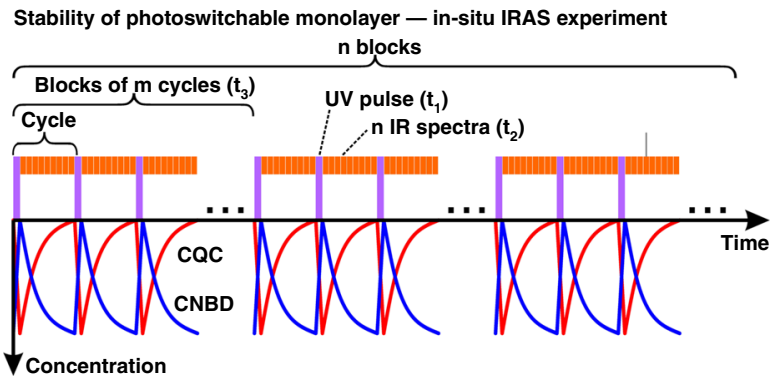

b
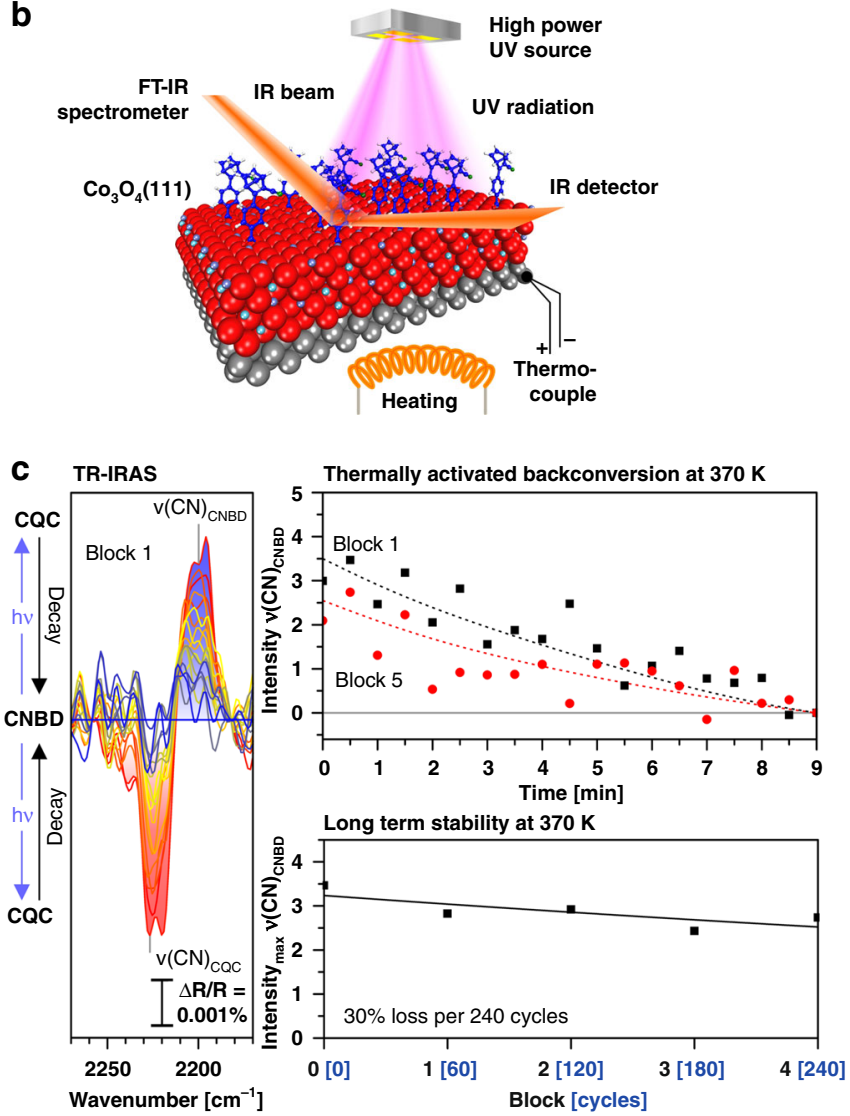

Fig. 5 Stability of the $\mathrm{CNBD}$ monolayer on $\mathrm{CO}_{3} \mathrm{O}_{4}(111)$ during (back-) conversion: a the experimental procedure of repeated photochemical conversion and thermally activated backconversion and (b) experimental setup used in the in-situ IRAS experiment; $\mathbf{c}$ IR spectra in the CN stretching frequency region of CNBD and CQC during photochemical conversion and thermally activated backconversion and development as a function of time, referenced to the anchored CNBD monolayer (black squares: block 1, red circles: block 5)

backconversion for CQC in solution (toluene), both in the protonated form (CQC, carboxylic acid) and in the deprotonated form (CQC, carboxylate). The activation barriers (CQC protonated: $108 \mathrm{~kJ} \cdot \mathrm{mol}^{-1}$, CQC deprotonated: $105 \mathrm{~kJ} \cdot \mathrm{mol}^{-1}$, see Supplementary Methods for details) are very close to the value found for the anchored monolayer. We conclude that, within the accuracy of our experiments, the anchoring reaction has no effect on the activation barrier for backconversion.

Stability of the photoswitchable monolayer. Finally, we tested the stability of the anchored CNBD/CQC monolayer during repeated energy storage and release cycles. The experimental procedure is illustrated in Fig. $5 \mathrm{a}, \mathrm{b}$. The anchored CNBD 
<smiles>CC(C)(C#N)CC(=O)c1ccc(I)cc1</smiles><smiles>N#CC1C2CCC1C2c1ccc(C(=O)O)cc1</smiles>

CQC
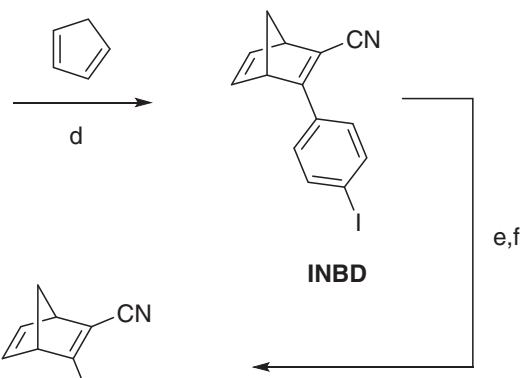

Fig. 6 Synthetic sequence for CNBD-CQC photoswitch couple. Conditions: (a) $\mathrm{POCl}_{3}, \mathrm{DMF}, 3 \mathrm{~h}$; (b) $\mathrm{I}_{2}, \mathrm{NH}_{3(\mathrm{aq})}, \mathrm{CHCl}_{3}, 3 \mathrm{~h}$; (c) $\mathrm{NaOH}(\mathrm{aq}), \mathrm{THF}, 4 \mathrm{~h}$; (d) chlorobenzene, $\mathrm{BHT}, 120^{\circ} \mathrm{C}, 20 \mathrm{~h}$; (e) $\mathrm{PrMgCl} \cdot \mathrm{LiCl}, \mathrm{THF},-41^{\circ} \mathrm{C}, 1 \mathrm{~h} ; \mathrm{CO}_{2(\mathrm{~g})},-41^{\circ} \mathrm{C}-\mathrm{RT}, 16 \mathrm{~h}$, followed by $\mathrm{HCl}_{(\mathrm{aq})}$

monolayer was exposed to pulses of UV light (60 s), followed by a decay period during which the backconversion was monitored by time-resolved IRAS (30 s per IR spectrum, 18 spectra). The photoconversion-decay sequence was repeated for a total duration of $50 \mathrm{~h}$ ( 300 cycles, $6000 \mathrm{IR}$ spectra). The data (averaged in blocks of 60 cycles to obtain better signal/noise ratio) are shown in Fig. $5 c$. Over the total duration of the experiment, we observed a decay of the band intensities of CQC and CNBD by $\sim 30 \%$. Assuming that the degradation follows an exponential behavior, this value corresponds to a loss of $0.15 \%$ per storage and release cycle. Comparing the peak height of the $v_{\mathrm{CNBD}}(\mathrm{CN})$ band in Fig. $5 \mathrm{c}$ to the monolayer spectrum (see Fig. $2 \mathrm{c}$ ), we estimate that $\sim 10 \%$ of the NBD monolayer is converted per UV pulse. This corresponds to a loss of $1.5 \%$ per converted NBD molecule, i.e., to a reversibility of $98.5 \%$ per energy storage and release cycle in the anchored film.

In conclusion, we assembled a solar-energy-storing organicoxide hybrid interface by anchoring a tailor-made photoswitch to an atomically defined oxide surface. The synthesized norbornadiene derivative 2-cyano-3-(4-carboxyphenyl)norbornadiene (CNBD) was linked to an atomically defined $\mathrm{Co}_{3} \mathrm{O}_{4}(111)$ surface by PVD under UHV conditions. We monitored the photoconversion of CNBD to the quadricyclane derivative CQC and the thermally activated backconversion in situ using a photochemical surface IR spectroscopy experiment. We determined the quantum efficiencies and showed that photoconversion is possible even in a single-anchored CNBD monolayer on $\mathrm{Co}_{3} \mathrm{O}_{4}(111)$. The anchored CNBD monolayer can be charged and discharged with high reversibility ( $98.5 \%$ reversibility), and the activation barrier for the thermally activated backconversion $\left(103 \pm 10 \mathrm{~kJ} \cdot \mathrm{mol}^{-1}\right)$ is not affected by the anchoring reaction. The results show that it is possible to assemble a monolayer of NBD photoswitches on a semiconducting oxide interface without affecting the functionality of the photoswitch. The model interface presented in this work will enable fundamental studies on well-defined hybrid interfaces for solar-energy storage and conversion. An important next step toward a functional energy storage device would be to trigger the energy-release process electrochemically instead of thermally.

\section{Methods}

Experimental setup. See Supplementary Methods for details.

Preparation of the $\mathrm{CO}_{3} \mathrm{O}_{4}$ (111) film. The ordered cobalt oxide thin films were prepared on an $\operatorname{Ir}(100)$ single crystal (MaTeck, purity $99.999 \%$ ) following an adapted method based on the procedure described by Heinz and Hammer ${ }^{27}$. The crystal was cleaned by cycles of $\mathrm{Ar}^{+}$ion bombardment (Linde 6.0). Annealing at $1370 \mathrm{~K}$ for 3 min led to formation of the $\operatorname{Ir}(100)-(5 \times 1)$ reconstructed surface which was checked by LEED. Successively, annealing in oxygen $\left(5 \times 10^{-8} \mathrm{mbar}\right.$, Linde 5.0$)$ at $1270 \mathrm{~K}$ for $3 \mathrm{~min}$ and cooling to $370 \mathrm{~K}$ in $\mathrm{O}_{2}$ yielded an $\operatorname{Ir}(100)-(2 \times 1) \mathrm{O}$ reconstructed surface as confirmed by LEED. Cobalt (Alfa Aesar, purity 99.95\%, $2 \mathrm{~mm}$ diameter rod) was deposited for $20 \mathrm{~min}$ at a sample temperature of $265 \mathrm{~K}$ in $\mathrm{O}_{2}$ atmosphere $\left(1 \times 10^{-6} \mathrm{mbar}\right)$ at a rate of $2 \AA / \mathrm{min}$ (Co metal equivalent) as determined by the QCM, yielding a $\mathrm{Co}_{3} \mathrm{O}_{4}$ film of $\sim 8 \mathrm{~nm}$ thickness. An ordered $\mathrm{Co}_{3} \mathrm{O}_{4}(111)$ film was formed in two annealing steps, at $520 \mathrm{~K}$ in oxygen atmosphere for $2 \mathrm{~min}$ and at $670 \mathrm{~K}$ in UHV for $10 \mathrm{~min}$, as confirmed by LEED ${ }^{25-27}$.

PVD of CNBD. CNBD was evaporated from a glass crucible loaded into a home built Knudsen cell. Prior to the first experiment, the system was separated from the main chamber by a gate valve, pumped by a separate high vacuum line, and baked for $24 \mathrm{~h}$. Before deposition, the evaporator was preheated to $370 \mathrm{~K}$ before the gate valve was opened to start the deposition.

During deposition, IR spectra were acquired at a rate of 1 spectrum $/ \mathrm{min}$ and a spectral resolution of $4 \mathrm{~cm}^{-1}$. The spectra were referenced to the background of the clean sample. For the temperature-programmed experiment, IR spectra were recorded at a rate of 1 spectrum $/ \mathrm{min}$ while heating the sample at a rate of $2 \mathrm{~K} / \mathrm{min}$. Damping of the signal with increasing temperature was compensated for, following a procedure described by $\mathrm{Xu}$ et $\mathrm{al}^{31}$, by normalization of the acquired spectra.

Photochemical conversion in UHV. All photochemical experiments in UHV were performed with a home-built high-intensity UV source in the vicinity of the sample. In brief, we used a high-power LED (Seoul Viosys, CUN6AF4A, 2.35 W), which yields a photon flux density of $1.68 \times 10^{-18} \mathrm{~cm}^{-2} \cdot \mathrm{s}^{-1}$ at a wavelength of 365 $\mathrm{nm}$, corresponding to a power density of $910 \mathrm{~mW} \cdot \mathrm{cm}^{-2}$. Further information can be found in the literature ${ }^{32}$. During all photoconversion experiments, the sample was cooled to $110 \mathrm{~K}$. The UV source was operated by an external power supply (TDK Lambda $Z+200$ ) triggered by the IR spectrometer (Bruker OPUS 7.2) or manually. For each sample, nine illumination steps were applied, such that the total illumination time was increasing exponentially $(0.01 \mathrm{~s}, 0.04 \mathrm{~s}, 0.16 \mathrm{~s}, 0.64 \mathrm{~s}, 2.56 \mathrm{~s}$, $10.24 \mathrm{~s}, 40.96 \mathrm{~s}, 163.84 \mathrm{~s}, 655.36 \mathrm{~s})$. After each illumination step, an IR spectrum was recorded with an acquisition time of $10 \mathrm{~min}$.

Thermally activated backconversion. Backconversion in the anchored CNBD film was recorded at three different temperatures, i.e., $350 \mathrm{~K}, 360 \mathrm{~K}, 370 \mathrm{~K}$. At each temperature, the sample was irradiated for $1 \mathrm{~min}$ followed by a decay period ( $89 \mathrm{~min}, 29 \mathrm{~min}, 19 \mathrm{~min})$ during which IR spectra were recorded ( $2 \mathrm{spectra} / \mathrm{min})$. The irradiation/decay cycles were repeated for $15 \mathrm{~h}$ at each temperature $(10,30,45$ cycles), and the data were accumulated over the cycles to improve the signal/noise ratio. Background spectra were taken before the measurement at each temperature step. The rate constants for backconversion were determined from exponential fits of the maximum peak heights between $v(\mathrm{CN})_{\mathrm{CNBD}}$ and $v(\mathrm{CN})_{\mathrm{CQC}}$.

Stability test. Stability of the anchored CNBD film was tested by applying 300 cycles of illumination $(1 \mathrm{~min})$ and decay $(9 \mathrm{~min})$ at $370 \mathrm{~K}$. IR spectra were recorded at a rate of 2 spectra/min (total acquisition time $50 \mathrm{~h}, 6000$ spectra in total). To improve the signal/noise ratio, the IR spectra were averaged over blocks of 60 spectra ( 5 blocks). The data were analyzed for the peak height of the $v(\mathrm{CN})_{\mathrm{CNBD}}$ band. The loss per cycle was estimated assuming an exponential decay.

Transmission IR data. Transmission IR spectra of CNBD and CQC were recorded in FTIR-grade $\mathrm{KBr}(\geq 99 \%$, Sigma Aldrich) using a FTIR spectrometer (Bruker VERTEX $80 \mathrm{v}$ ) at a spectral resolution of $2 \mathrm{~cm}^{-1}$ (acquisition time $1 \mathrm{~min}$ ). For the spectrum of CQC, the CNBD sample was irradiated in an external cell by UV light (Seoul Viosys, CUN6AF4A, $365 \mathrm{~nm}$, distance to sample $2 \mathrm{~cm}$, illumination time $10 \mathrm{~min}$ ). 
Synthesis. The molecular photoswitch CNBD was synthesized in a multistep sequence starting from commercially available 4 -iodoacetophenone. This included an initial three-step transformation of the acetyl functionality to the corresponding propiolonitrile. Direct conjugation of the nitrile to the acetylene activated $\mathbf{1}$ toward a Diels-Alder $[4+2 \pi]$ cycloaddition reaction with cyclopentadiene to afford INBD in excellent yield. Knöchel conditions were finally used to promote an iodine-magnesium exchange and trapping of the carbanion with carbon dioxide generated CNBD (see Fig. 6).

All intermediates and products were characterized by ${ }^{1} \mathrm{H}$ NMR and ${ }^{13} \mathrm{C}$ NMR. The final products CNBD and CQC were characterized by UV-vis spectroscopy, including quantum yields for the photochemical conversion. Thermodynamic and kinetic data regarding the backconversion from CNBD to CQC were determined by differential scanning calorimetry (DSC) and kinetic UV-vis studies. The structure of CNBD was determined by single-crystal X-ray crystal structural analysis. All details of synthesis and characterization are given in the Supplementary Methods.

DFT calculations. See Supplementary Methods for details.

\section{Data availability}

All experimental data are available from the corresponding author upon request.

Received: 29 January 2019 Accepted: 1 May 2019

Published online: 03 June 2019

\section{References}

1. Lewis, N. S. \& Nocera, D. G. Powering the planet: chemical challenges in solar energy utilization. Proc. Natl Acad. Sci. USA 103, 15729 (2006).

2. International Energy Agency. Technology Roadmap - Solar Thermal Electricity 2014 https://www.iea.org/publications/freepublications/publication/ TechnologyRoadmapSolarThermalElectricity_2014edition.pdf (2014).

3. Kucharski, T. J. et al. Templated assembly of photoswitches significantly increases the energy-storage capacity of solar thermal fuels. Nat. Chem. 6, 441 (2014).

4. Kucharski, T. J., Tian, Y., Akbulatov, S. \& Boulatov, R. Chemical solutions for the closed-cycle storage of solar energy. Energy Environ. Sci. 4, 4449-4472 (2011).

5. Krekiehn, N. R. et al. UV/Vis spectroscopy studies of the photoisomerization kinetics in self-assembled azobenzene-containing adlayers. Langmuir 31, 8362-8370 (2015).

6. Dauben, W. G. \& Cargill, R. L. Photochemical transformations-VIII: the isomerization of $\Delta 2,5$-bicyclo[2.2.1] heptadiene to quadricyclo[2.2.1.02,6.03,5] heptane (quadricyclene). Tetrahedron 15, 197-201 (1961).

7. Schwendiman, D. P. \& Kutal, C. Catalytic role of copper(I) in the photoassisted valence isomerization of norbornadiene. J. Am. Chem. Soc. 99, 5677-5682 (1977).

8. Cuppoletti, A., Dinnocenzo, J. P., Goodman, J. L. \& Gould, I. R. Bond-coupled electron transfer reactions: photoisomerization of norbornadiene to quadricyclane. J. Phys. Chem. B. 103, 11253-11256 (1999).

9. Grutsch, P. A. \& Kutal, C. Charge-transfer sensitization of the valence photoisomerization of norbornadiene to quadricyclene by an orthometalated transition-metal complex. J. Am. Chem. Soc. 108, 3108-3110 (1986).

10. Tung, C.-H., Zhang, L.-P., Li, Y., Cao, H. \& Tanimoto, Y. Benzophenoneinitiated photoisomerization of the norbornadiene group in a benzophenone -steroid-norbornadiene system via long-distance intramolecular triplet energy transfer. J. Phys. Chem. 100, 4480-4484 (1996).

11. Maruyama, K., Terada, K., Naruta, Y. \& Yamamoto, Y. Photoisomerization of norbornadiene to quadricyclane in the presence of copper(I)-nitrogen ligand catalysts. Chem. Lett. 9, 1259-1262 (1980).

12. Dreos, A. et al. Liquid norbornadiene photoswitches for solar energy storage. Adv. Energy Mater. 8, 1703401 (2018).

13. Wang, Z. et al. Macroscopic heat release in a molecular solar thermal energy storage system. Energy Environ. Sci. 12, 187-193 (2019).

14. Moth-Poulsen, K. et al. Molecular solar thermal (MOST) energy storage and release system. Energy Environ. Sci. 5, 8534-8537 (2012).

15. Yoshida, Z.-i New molecular energy storage systems. J. Photochem. 29, 27-40 (1985).

16. Bren, V. A., Alexander, D. D., Vladimir, I. M. \& Chernoivanov, V. A. Norbornadiene-quadricyclane - an effective molecular system for the storage of solar energy. Russ. Chem. Rev. 60, 451 (1991).

17. Vessally, E. \& Aryana, S. Maximizing the solar energy storage of the four substituted norbornadiene-quadricyclane system: DFT calculations. Russ. J. Phys. Chem. A 90, 136-143 (2016).

18. Quant, M. et al. Low molecular weight norbornadiene derivatives for molecular solar-thermal energy storage. Chem.: Eur. J. 22, 13265-13274 (2016).
19. Mansø, M. et al. Molecular solar thermal energy storage in photoswitch oligomers increases energy densities and storage times. Nat. Commun. 9, 1945 (2018).

20. Jorner, K. et al. Unraveling factors leading to efficient norbornadiene-quadricyclane molecular solar-thermal energy storage systems. J. Mater. Chem. A 5, 12369-12378 (2017).

21. Jevric, M. et al. Norbornadiene-based photoswitches with exceptional combination of solar spectrum match and long-term energy storage. Chem.: Eur. J. 24, 12767-12772 (2018).

22. Brummel, O. et al. Energy storage in strained organic molecules: (spectro) electrochemical characterization of norbornadiene and quadricyclane. ChemSusChem 9, 1424-1432 (2016).

23. Brummel, O. et al. Photochemical energy storage and electrochemically triggered energy release in the norbornadiene-quadricyclane system: UV photochemistry and IR spectroelectrochemistry in a combined experiment. J. Phys. Chem. Lett. 8, 2819-2825 (2017).

24. Schwarz, M. et al. Anchoring of a carboxyl-functionalized norbornadiene derivative to an atomically defined cobalt oxide surface. J. Phys. Chem. C 121, 11508-11518 (2017).

25. Meyer, W., Biedermann, K., Gubo, M., Hammer, L. \& Heinz, K. Surface structure of polar $\mathrm{Co}_{3} \mathrm{O}_{4}(111)$ films grown epitaxially on $\operatorname{Ir}(100)-(1 \times 1)$. J. Phys. Condens. Matter 20, 265011 (2008).

26. Biedermann, K., Gubo, M., Hammer, L. \& Heinz, K. Phases and phase transitions of hexagonal cobalt oxide films on $\operatorname{Ir}(100)-(1 \times 1)$. J. Phys. Condens. Matter 21, 185003 (2009).

27. Heinz, K. \& Hammer, L. Epitaxial cobalt oxide films on $\operatorname{Ir}(100)$ - the importance of crystallographic analyses. J. Phys. Condens. Matter 25, 173001 (2013).

28. Chen, J., Wu, X. \& Selloni, A. Electronic structure and bonding properties of cobalt oxide in the spinel structure. Phys. Rev. B 83, 245204 (2011).

29. $\mathrm{Xu}, \mathrm{T}$. et al. Structure-dependent anchoring of organic molecules to atomically defined oxide surfaces: phthalic acid on $\mathrm{Co}_{3} \mathrm{O}_{4}(111), \mathrm{CoO}(100)$, and $\mathrm{CoO}$ (111). Chem.: Eur. J. 22, 5384-5396 (2016).

30. Werner, K. et al. Functionalized porphyrins on an atomically defined oxide surface: anchoring and coverage-dependent reorientation of MCTPP on $\mathrm{Co}_{3} \mathrm{O}_{4}(111)$. J. Phys. Chem. Lett. 7, 555-560 (2016).

31. Xu, T. et al. The surface structure matters: thermal stability of phthalic acid anchored to atomically-defined cobalt oxide films. Phys. Chem. Chem. Phys. 18, 10419-10427 (2016)

32. Schwarz, M. et al. A simple high-intensity UV-photon source for photochemical studies in UHV: application to the photoconversion of norbornadiene to quadricyclane. Rev. Sci. Instrum. 90, 024105 (2019).

\section{Acknowledgements}

This project was supported by the Deutsche Forschungsgemeinschaft (DFG). Additional support by the DFG was provided within the Research Unit FOR 1878 "funCOS Functional Molecular Structures on Complex Oxide Surfaces". K.M.P., M.J., A.U.P., and Z.W. would like to thank the Knut \& Alice Wallenberg Foundation (KAW), the Swedish foundation for Strategic Research (SSF) and K.M.P. would like to thank the European Research Council (ERC) for financial support. A.G. and L.F. would like to thank the Free State of Bavaria for the support provided within the research network "Solar Technologies go Hybrid" (SolTech).

\section{Author contributions}

C.S. performed the photochemical IRAS experiments and analyzed the data with the help of C.H., M.S., F.W. and M.K. M.J. performed the synthesis of the new compounds CNBD and CQC and A.U.P., Z.W. and C.J.S. were involved in the characterization of CNBD and CQC. K.M.P. supervised the synthetic work and was involved in the analysis of the data. L.F. performed the DFT calculations and A.G. supervised the theoretical work. O.B. and J.L. supervised the IR spectroscopy experiments and were involved in the analysis of the data. C.S. and J.L. prepared the paper with the help of the other coauthors.

\section{Additional information}

Supplementary Information accompanies this paper at https://doi.org/10.1038/s41467019-10263-4.

Competing interests: The authors declare no competing interests.

Reprints and permission information is available online at http://npg.nature.com/ reprintsandpermissions/

Journal peer review information: Nature Communications thanks Katharina $\mathrm{Al}$ Shamery, Ulrike Diebold and the other, anonymous, reviewer(s) for their contribution to the peer review of this work. Peer reviewer reports are available.

Publisher's note: Springer Nature remains neutral with regard to jurisdictional claims in published maps and institutional affiliations. 
(c) (i) Open Access This article is licensed under a Creative Commons Attribution 4.0 International License, which permits use, sharing, adaptation, distribution and reproduction in any medium or format, as long as you give appropriate credit to the original author(s) and the source, provide a link to the Creative Commons license, and indicate if changes were made. The images or other third party material in this article are included in the article's Creative Commons license, unless indicated otherwise in a credit line to the material. If material is not included in the article's Creative Commons license and your intended use is not permitted by statutory regulation or exceeds the permitted use, you will need to obtain permission directly from the copyright holder. To view a copy of this license, visit http://creativecommons.org/ licenses/by/4.0/.

(C) The Author(s) 2019 